\title{
Severe geomagnetic storms and Forbush decreases: interplanetary relationships reexamined
}

\author{
R. P. Kane \\ Instituto Nacional de Pesquisas Espaciais, C.P. 515, 12245-970 - São José dos Campos, SP, Brazil \\ Received: 22 June 2009 - Revised: 27 November 2009 - Accepted: 30 November 2009 - Published: 9 February 2010
}

\begin{abstract}
Severe storms (Dst) and Forbush decreases (FD) during cycle 23 showed that maximum negative Dst magnitudes usually occurred almost simultaneously with the maximum negative values of the $B_{z}$ component of interplanetary magnetic field $\mathrm{B}$, but the maximum magnitudes of negative Dst and $B_{z}$ were poorly correlated (+0.28). A parameter $B_{z}(\mathrm{CP})$ was calculated (cumulative partial $B_{z}$ ) as sum of the hourly negative values of $B_{z}$ from the time of start to the maximum negative value. The correlation of negative Dst maximum with $B_{z}(\mathrm{CP})$ was higher $(+0.59)$ as compared to that of Dst with $B_{z}$ alone (+0.28). When the product of $B_{z}$ with the solar wind speed $V$ (at the hour of negative $B_{z}$ maximum) was considered, the correlation of negative Dst maximum with $V B_{z}$ was +0.59 and with $V B_{z}(\mathrm{CP})$, 0.71 . Thus, including $V$ improved the correlations. However, ground-based Dst values have a considerable contribution from magnetopause currents (several tens of $\mathrm{nT}$, even exceeding $100 \mathrm{nT}$ in very severe storms). When their contribution is subtracted from Dst(nT), the residue Dst* representing true ring current effect is much better correlated with $B_{z}$ and $B_{z}(\mathrm{CP})$, but not with $V B_{z}$ or $V B_{z}(\mathrm{CP})$, indicating that these are unimportant parameters and the effect of $V$ is seen only through the solar wind ram pressure causing magnetopause currents. Maximum negative Dst (or Dst*) did not occur at the same hour as maximum FD. The time evolutions of Dst and FD were very different. The correlations were almost zero. Basically, negative Dst (or Dst*) and FDs are uncorrelated, indicating altogether different mechanism.
\end{abstract}

Keywords. History of geophysics (Solar-planetary relationships) - Interplanetary physics (Cosmic rays) - Solar physics, astrophysics, and astronomy (Flares and mass ejections)

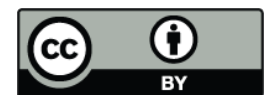

Correspondence to: R. P. Kane (kane@dge.inpe.br)

\section{Introduction}

In data from ionization chambers, Forbush (1937) and Hess and Demmelmair (1937) observed decreases in the cosmic ray count rate, which typically lasted for a week. Simpson (1954) hinted that the origin of these decreases was probably in the interplanetary medium. Later, these were found to be associated with geomagnetic storms and their drivers ICMEs (Interplanetary continuations of the solar Coronal Mass Ejections CMEs), magnetic clouds, as well as corotating shock fronts.

There are two basic types of Forbush decreases (hereafter abbreviated as FDs, more details in the review Cane, 2000): (a) Non-recurrent decreases which are caused by transient interplanetary events related to mass ejections from the Sun, are marked by a sudden onset, reach their maximum depression in about a day, and are characterized by a gradual recovery; and (b) Recurrent decreases, which have a gradual onset and are symmetric in profile; and tend to be associated with corotating high speed solar wind streams (Lockwood, 1971; Iucci et al., 1979a). Though geomagnetic disturbances and FDs have a common origin in interplanetary space, namely encountering a strong interplanetary structure (blob), the magnitudes of geomagnetic disturbances and FDs are not proportional to each other (e.g., Kane, 1977). Geomagnetic disturbances occur by the Dungey (1961) mechanism, where reconnection occurs at the daytime magnetopause between the terrestrial magnetic field and the southward $B_{z}$ component of the interplanetary field. When the field lines are swept back in the geomagnetic tail, a neutral point is formed through which the solar wind gets an entry into the magnetosphere. Low energy particles spiral around the stretched geomagnetic field lines and impinge on the terrestrial atmosphere in the polar regions, causing enhanced aurora. Higher energy particles rush towards the Earth but are diverted around the Earth (Fleming's right hand rule) in circular orbits in the equatorial plane, forming a ring current

Published by Copernicus Publications on behalf of the European Geosciences Union. 
at several earth radii, which causes large geomagnetic field reductions. These reductions in the terrestrial magnetic field strength are measured by the Dst (disturbance storm time index, Sugiura, 1964). Measurements of Dst and FDs indicate some commonality in their interplanetary sources; however, there are significant differences in the exact evolution of each measure (Cane, 2000). In general, both increase with increasing IMF (interplanetary magnetic field $\boldsymbol{B}$ ) and SW (solar wind) velocity $V$. An increase in SW density $N$ and speed $V$ affects the magnetosphere but not CR intensity. The most important difference is that FDs are governed by the conditions in a large volume of the heliospheric region, while Dst variation depends on the local situation in the magnetotail near the Earth.

All short-term CR decreases have been historically called Forbush decreases. However, some workers are using this term only for the non-recurrent events with a sudden onset and gradual recovery. Such "classical" Forbush decreases generally have two steps. As described in Cane (2000), Coronal Mass Ejections (CMEs) are plasma eruptions from the solar atmosphere involving previously closed field regions, which are expelled into the interplanetary medium. The ICMEs (interplanetary CMEs), termed as "ejecta", may generate shocks. When Earth enters such a "shock followed by ejecta" combination, the first step in the classical Forbush decrease is due to entry in the shock. The second step was attributed by different workers to different features. Barnden (1973) attributed it to the discontinuity marking entry from the shock into the ejecta. Iucci et al. (1986) attributed it to the magnetic field increase comprised of the post-shock compression region and the ejecta. Nagashima et al. (1990) attributed it to the stratified structure of disturbed and undisturbed magnetic fields. Cane (2000) illustrated the largescale structure of an ejecta and the associated shock and further, how the cosmic ray response is related to the path through the ensemble. She concluded that CME-related CR decreases are of three basic types; those caused by a shock and ejecta, by a shock only, and by an ejecta only. A majority ( $>80 \%$ ) of short-term CR decreases $>4 \%$ are of the two-step (shock plus ejecta) type (Cane et al., 1996).

Relations between FD magnitudes and the solar wind IMF (Barden, 1973; Iucci et al., 1979a, b; Burlaga et al., 1984; Cane, 1993) and solar wind velocity (Iucci et al., 1979a, b; Belov and Ivanov, 1997) have been studied since long, but the magnitude of the FD and the interplanetary magnetic field (IMF) or the proton velocity $V$ alone have not correlated well. Several studies have shown improved correlations when both the field $\boldsymbol{B}$ and speed $V$ are simultaneously considered. Using data for 695 events, Belov et al. (2001) correlated FD magnitude with the product $H_{\max } V_{\max }$ and found a correlation of 0.70 , while correlation with $H_{\max }$ (i.e., magnetic field $B_{\max }$ ) alone was 0.66 and with $V_{\max }$ alone was only 0.22. (This fits several theoretical models, e.g., Wibbernez et al., 1998, and references therein). For the relationship between FD magnitudes and geomagnetic activity, using more than 1400 events during 1978-1996, Belov et al. (2001) obtained a correlation of 0.42 between FD magnitudes and the maximum geomagnetic disturbance index Kp (correlations with Dst were smaller). On the average, very small FDs $(\leq 1 \%)$ were reported to be associated with quiet and unsettled geomagnetic conditions $(\mathrm{Kp} \leq 3)$. Even up to $\mathrm{Kp}=5$, the associated FDs were small $(\leq 1.5 \%)$. Large FDs were associated with $\mathrm{Kp} \geq 8$. The FDs of July 2005 and August 2005 have been discussed in detail by Papaioannou et al. (2009a, b). Earlier, Cane et al. (1996) studied 30 years (1964-1994) of neutron monitor data to understand the principle mechanism causing short-term CR decreases seen at Earth. Examining the characteristics of associated low energy $(<200 \mathrm{MeV})$ particle enhancements with the neutron monitor data, they identified the responsible solar wind disturbance structures for 153 of the 180 events of $\geq 4 \%$ $\mathrm{CR}$ decreases. The classes of solar wind structures were: (1) shock plus ejecta, (2) shock, (3) shock plus ejecta less energetic than class 1, (4) complex event including a corotating high speed stream. Recently, Kudela and Brenkus (2004) have presented for 1982-2002 two lists of strong events, in their Table 1 for CR decreases at the location Lomnický Stit (49.2 N, 20.2 E) $\geq 6 \%$, and in their Table 2 for Dst $<-200 \mathrm{nT}$. Their events before 1994 tallied with those of Cane et al. (1996).

The purpose of the present communication is to reexamine the severe Dst events for their relationships with FDs and interplanetary parameters, first for cycle 23 and then for some selected very severe Dst events and very large FDs in the previous cycles 19-22.

\section{Data}

Data were obtained from Cane et al. (1996) (henceforth called Cane events) and from the NGDC website http://spidr. ngdc.noaa.gov/spidr/index.jsp. The Cane events are ICME events classified as to whether these had ejecta (drivers) or shocks or ejecta with shocks. From these only those are considered which were associated with occurrences of $\geq 4 \%$ CR decreases during each year of 1965-1994 (thus, very weak ICMEs are mostly eliminated). For cosmic rays, data used are for the neutron monitor at Climax (northern middle latitude).

\section{Summary of correlations for the Cane events}

The correlations obtained for the Cane events up to 1994 are summarized in Table 1. We consider correlations below 0.30 as low, $0.30-0.59$ as moderate and exceeding 0.60 as high.

The following may be noted in Table 1 .

1. The correlations for classes 1, 2, 3, 4 are not similar. Where CR are involved, class 1 (shocks plus ejecta) 
Table 1. Correlations of $C R$ decreases and Dst depressions with interplanetary $V$, magnetic field components $B, B_{z}$, and various combinations of the same.

\begin{tabular}{lrrrr}
\hline & Class 1 & Class 2 & Class 3 & Class 4 \\
\hline CR versus $V$ & 0.57 & 0.37 & 0.41 & -0.14 \\
Dst versus $V$ & -0.21 & -0.3 & -0.52 & -0.68 \\
& & & & \\
CR versus total $B$ & 0.22 & 0.02 & 0.08 & 0.02 \\
Dst versus total $B$ & -0.26 & -0.23 & -0.53 & -0.6 \\
& & & & \\
CR versus $B_{z}$ & -0.28 & 0.01 & -0.24 & -0.24 \\
Dst versus $B_{z}$ & 0.47 & 0.64 & 0.78 & 0.65 \\
CR versus $V B$ & & & & \\
Dst versus $V B_{z}$ & 0.46 & 0.17 & 0.33 & -0.08 \\
& 0.67 & 0.72 & 0.91 & 0.82 \\
CR versus $V$ & & & & \\
CR versus $(V-V o)$ & 0.63 & 0.48 & 0.41 & -0.22 \\
CR versus $(V / V o)$ & 0.59 & 0.61 & 0.37 & -0.13 \\
& 0.41 & 0.58 & 0.24 & -0.02 \\
CR versus total $B$ & & & & \\
CR versus $(B-B o)$ & 0.19 & 0.03 & 0.22 & -0.09 \\
CR versus $(B / B o)$ & 0.12 & 0.07 & 0.3 & -0.02 \\
& -0.2 & 0.21 & 0.62 & -0.03 \\
CR versus $V B$ & & & & \\
CR versus $(V-V o)(B-B o)$ & 0.44 & 0.22 & 0.37 & -0.17 \\
CR versus $(V / V o)(B / B o)$ & 0.46 & 0.36 & 0.4 & -0.07 \\
CR versus $V B t$ & -0.07 & 0.34 & 0.6 & -0.01 \\
CR versus $(V-V o)(B-B o)(V-V o)(B-B o) t$ & 0.65 & 0.19 & 0.47 & 0.18 \\
CR versus $(V / V o)(B / B o)(V / V o)(b / B o) t$ & 0.66 & 0.28 & 0.46 & 0.13 \\
\hline & 0.32 & 0.29 & 0.72 & 0.2 \\
\hline
\end{tabular}

shows at least moderate correlations, but class 4 (corotating streams) shows very low correlations. Where Dst is involved, class 4 shows some high correlations.

2. Correlations of CR decreases with interplanetary parameters $V, \boldsymbol{B}, B_{z}$ and $V B$ are low, indicating no straightforward relationship. In contrast, Dst has a good correlation with $B_{z}$ and $V B_{z}$.

3. Following indications of the model of Wibbernez et al. (1998), for CR decreases, the parameters $(V / V o)$ were considered in place of $V$, and $(B / B o)$ in place of $B$, where $V o$ and $B o$ are the base values before the start of the interplanetary event. These do not seem to make much difference. The correlations are still low. Thus, the role of the diffusion coefficient mentioned by Wibbernez et al. (1998) may be very important, but it cannot be determined.

4. In each storm, the duration " $t$ " can be estimated as the interval during which the hourly values of the total magnetic field $\boldsymbol{B}$ were high. Addition of the parameter " $t$ ", the duration for which the storm lasted, seems to im- prove the correlations for CR decreases for class 1, indicating a relation with the overall dimensions of the interplanetary structure.

Some of these average features will now be checked for severe Dst storms and FDs in Cycle 23.

\section{Severe geomagnetic storms in cycle 23}

Figures 1-4 show plots for 1996-2005 (cycle 23) for interplanetary parameters wind speed $V$, the magnetic total field $\boldsymbol{B}$ and its $B_{z}$ component, and for cosmic ray intensity at Climax, and the geomagnetic Dst. Only events where Dst depression was severe (numerically $>200 \mathrm{nT}$ ) are shown. Details are given in Table 2. The following may be noted:

\subsection{Relationship of Dst with $B_{z}$}

1. In all figures, a striking feature is the matching of negative Dst with interplanetary negative $B_{z}$, a feature wellknown since long (e.g., Kane, 1977; Gonzalez et al., 1994; Gonzalez and Echer 2005). 
Table 2. Details of seventeen severe Dst events (numerically $>200 \mathrm{nT}$ ) in cycle 23 .

\begin{tabular}{|c|c|c|c|c|c|c|c|c|c|c|}
\hline Event & Year & $\begin{array}{c}\text { Dst (min) } \\
\text { nT }\end{array}$ & $\begin{array}{c}B_{z}(\min ) \\
\mathrm{nT}\end{array}$ & $\begin{array}{l}\text { Cumulativ } \\
\text { upto } B_{Z} \text { (min) } \\
\text { nT }\end{array}$ & $\begin{array}{l}B_{z} \\
\text { Total } \\
\text { nT }\end{array}$ & $\begin{array}{c}B(\max ) \\
\mathrm{nT}\end{array}$ & $\begin{array}{c}B-B o \\
\text { nT }\end{array}$ & $\mathrm{FD}(\%)$ & $\begin{array}{c}V \\
\mathrm{~km} \mathrm{~s}^{-1}\end{array}$ & $N$ \\
\hline 4 May & 1998 & -205 & -29.6 & -92 & -69 & 37.9 & 29.9 & -4.5 & 867 & 29.6 \\
\hline $25 \mathrm{Sep}$ & 1998 & -207 & -17.9 & -161 & -29 & 19.5 & 9.5 & -7.5 & 766 & 10.3 \\
\hline $21-22$ Oct & 1999 & -231 & -30.7 & -149 & -140 & 35.6 & 25.6 & -2.3 & 529 & 12.1 \\
\hline 6-7 Apr & 2000 & -288 & 27.5 & -184 & -123 & 30.3 & 20.3 & -2.6 & & \\
\hline $15-16 \mathrm{Jul}$ & 2000 & -301 & & & & & & -11.5 & & \\
\hline $12 \mathrm{Aug}$ & 2000 & -235 & -28.9 & -214 & -87 & 33.3 & 23.3 & -4 & & \\
\hline 17-18 Sep & 2000 & -201 & -25 & -48 & -34 & 38.3 & 28.3 & -8.3 & & \\
\hline $31 \mathrm{Mar}$ & 2001 & -358 & -44.9 & -135 & -99 & 46.8 & 36.8 & -4.2 & 694 & 15.7 \\
\hline 11-12 Apr & 2001 & -256 & -20.8 & -144 & -102 & 33.1 & 23.1 & -11.2 & 730 & 12.2 \\
\hline 5-6 Nov & 2001 & -277 & & & & & & -10.3 & & \\
\hline 24 Nov & 2001 & -213 & & & & & & -10.1 & & \\
\hline 29 Oct & 2003 & -363 & -24.5 & -160 & -73 & 47.2 & 37.2 & -25.7 & 1300 & \\
\hline $30 \mathrm{Oct}$ & 2003 & -388 & -29.2 & -87 & -66 & 37.8 & 27.8 & -21.2 & 1300 & \\
\hline 20-21 Nov & 2003 & -472 & -45.6 & -315 & -122 & 55.8 & 45.8 & -5.2 & 589 & 25 \\
\hline 7-8 Nov & 2004 & -373 & -43.8 & -315 & -194 & 38.5 & 28.5 & -7.9 & 635 & 6.6 \\
\hline 15 May & 2005 & -263 & -37 & -73 & -51 & 53.5 & 43.5 & -10.1 & 892 & 11 \\
\hline $24 \mathrm{Aug}$ & 2005 & -216 & -39 & -69 & -39 & 49 & 39 & -8.1 & 621 & 19.4 \\
\hline
\end{tabular}

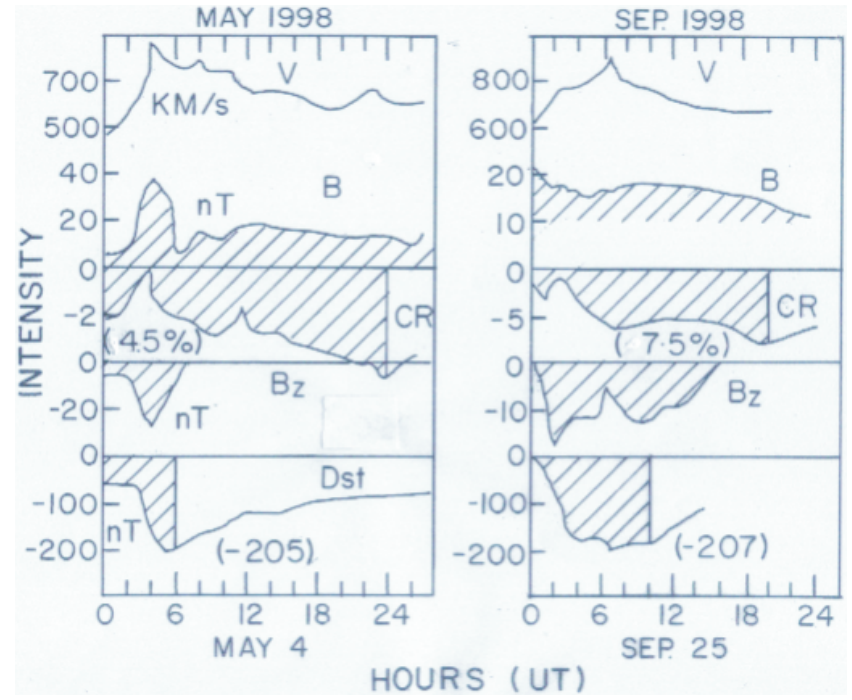

Fig. 1. Plots of hourly values of interplanetary $V, B, B_{z}$, Cosmic ray intensity (CR) at Climax and geomagnetic disturbance index Dst, for the two events 4 May 1998 and 25 September 1998 (ND means no data).

2. However, the correlation between the maximum magnitudes of negative Dst and negative $B_{z}$ was only +0.28 , in contrast to $0.47-0.78$ mentioned in Table 1 . This could be because there in Cane events, a large range of values was involved ( $\mathrm{FD} \geq 4 \%$ onwards). There were many pairs where low values of Dst were associated with low values of FDs, and with high values at the other end (some large Dst values associated with some large FDs), the contrast gave higher correlations. Now, the ranges are lesser (Dst only above $200 \mathrm{nT}$ ) and finer discrepancies get magnified and yield lower correlations.

3. Since the process of ring current intensification is more like a charging of an electrical condenser where fluctuations in the input are wiped out and only cumulative charge is registered, we calculated two more parameters namely, $B_{z}(\mathrm{CP})$, i.e., cumulative partial $B_{z}$, defined as the sum of successive negative hourly $B_{z}$ values from the time it turned negative to the time when it attained maximum negative value, and $B_{z}(\mathrm{CT})$, i.e., cumulative total $B_{z}$, defined as the sum of successive negative hourly $B_{z}$ from the time it turned negative to the time when negative values ended. The correlation of Dst with $B_{z}(\mathrm{CP})$ was substantial, +0.59 , a great improvement over the correlation with $B_{z}$ alone $(+0.28)$. The correlation with $B_{z}(\mathrm{CT})$ was +0.48 , indicating that the negative $B_{z}$ values after the $B_{z}$ negative peak occurred were not of much consequence.

4. In Table 1 , not just $B_{z}$ but its product $V B_{z}$ showed better correlations. For cycle 23, the correlation of Dst with $V B_{z}$ was +0.59 (better than +0.28 with $B_{z}$ alone). Also, the correlation of Dst with $V B_{z}(\mathrm{CP})$ was still more, +0.71 . Thus, using cumulative $B_{z}$ and its product with $V$ improved correlations considerably (Belov et al., 2001).

5. Adding the number density $N$, the correlation of Dst with $N V B_{z}(\mathrm{CP})$ was +0.68 , less than the +0.71 with 


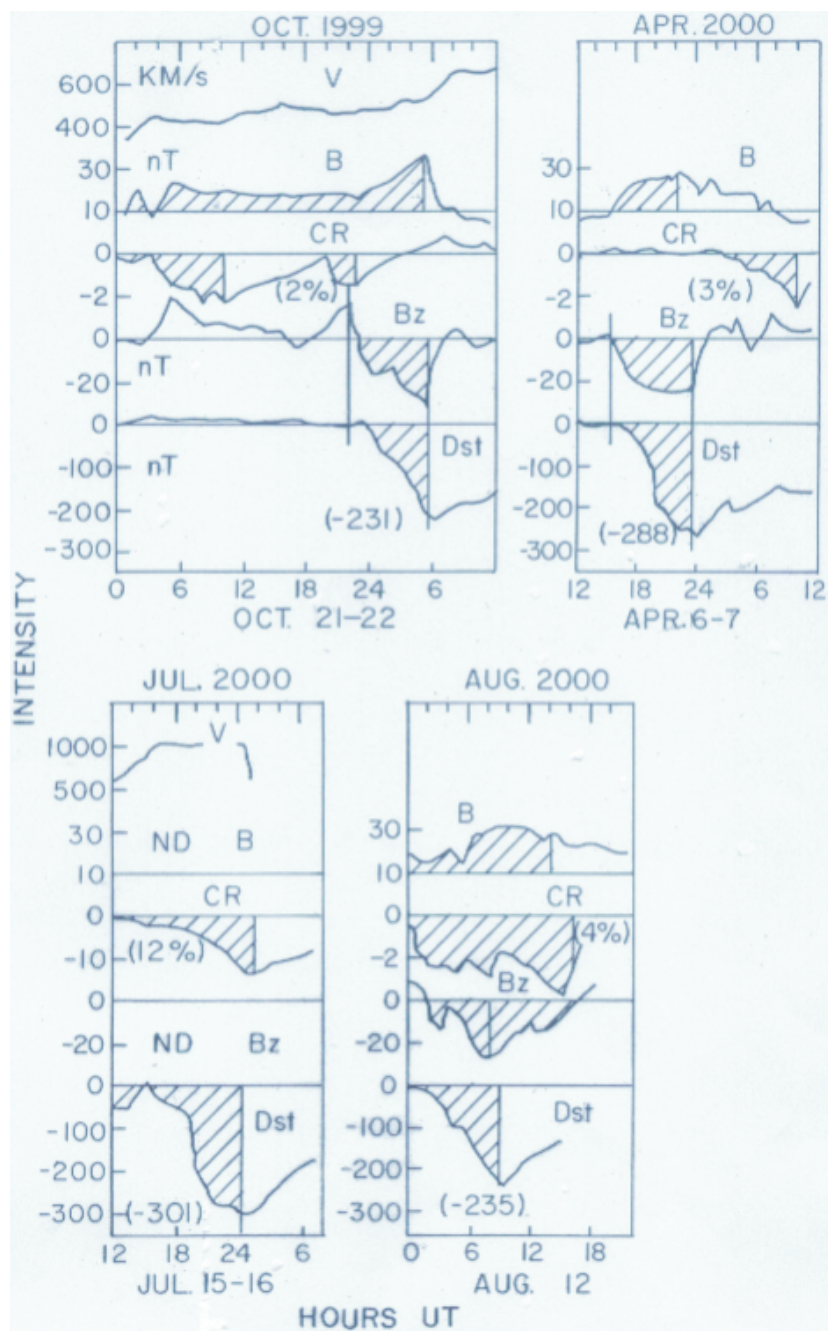

Fig. 2. Same as Fig. 1, for the four events 21-22 October 1999, 6-7 April 2000, 15-16 July 2000 and 12 August 2000.

$V B_{z}(\mathrm{CP})$. Thus, the solar wind number density does not seem to matter.

Figure 5 shows the plots of Dst versus (a) $B_{z}$ alone, corr. +0.28 , (b) $B_{z}(\mathrm{CP})$, corr. +0.59 , (c) $V B_{z}$, corr. +0.50 and (d) $V B_{z}(\mathrm{CP})$, corr. +0.71 .

\subsection{Relationship of Dst with FD}

Figure 6 shows a plot of FD magnitudes versus Dst for the seventeen events in cycle 23 (small dots). The scatter is large and the correlation is poor $(+0.31)$. There is only one indication that very large Dst (more than $350 \mathrm{nT}$ ) is associated with some very large FDs (exceeding 20\%) namely that during the Halloween events of 29-30 October 2003 (marked H). In some other cases, FDs of $\sim 10 \%$ were associated with Dst in the range 200-300 nT. (Incidentally, the low correlation of +0.31 is partly because of the restricting Dst to $<-200 \mathrm{nT}$.
If this restriction is removed, all FDs of cycle 23 has a higher correlation of +0.55$)$.

\subsection{Relationship of FD with interplanetary magnetic field $B$}

The correlatons of FDs with total magnetic field $\boldsymbol{B}$ and the excess $B-B o$ were -0.18 and -0.17 , very poor indeed. (according to Belov et al. (2001), the correlation is better if all FD events of cycle 23 are considered). With $V$, the correlation was high (0.90) indicating that in general, a fast, big interplanetary blob was likely to give a large FD. With number density $N$, the correlation was -0.40 , but this is unreliable as many values were missing, notably during the 29-30 October Halloween event when $N$ values were unreliable (abnormally low, Skoug et al., 2004. Also note that in view of the uncertainties and interruptions in the data reception during the Halloween events of 29-30 October 2003, we have used approximate values, namely, $-B_{z}(\min )=50 \mathrm{nT}, B(\max )=60 \mathrm{nT}$, $V=2000 \mathrm{~km} \mathrm{~s}^{-1}$ and $N=15$ for both these events). The correlation with $V B$ was reasonably high (0.78), mainly because of $V$. If the duration " $t$ " of $B$ was considered, the correlations with $B t$ and $V B t$ were almost the same or slightly inferior to those of $B$ and $V B$, but this was mainly because the " $t$ " was almost the same $(\sim 20 \mathrm{~h})$ for all the events. Judging " $t$ " is considerably subjective.

A disconcerting feature is that the $B$ maxima do not tally with the FD minima and often, their evolutions (variations with time) are dissimilar. Often when $B$ recovers to prestorm level, FD continues. The start of increased $B$ and FD is generally simultaneous, but the end is not similar. Sometimes, FD shows two events within a few hours, but $B$ does not show a double hump. Since $B$ is the only parameter responsible for deviating cosmic ray trajectories, we suspect that the $B$ noted as snapshot at the satellite location does not represent fully the overall large scale structure of $B$ in the blob.

\section{Severe geomagnetic storms and FDs in previous cy- cles 19-22}

The plots for the six largest geomagnetic storms (Dst numerically $>350 \mathrm{nT}$ ) in the past cycles 19-22 are shown in Fig. 7 (noted in Table 3). The six pairs of Dst and FD are plotted in Fig. 6 as open circles. Being all for high Dst values $(>359 \mathrm{nT})$, these are in the right end part, but the corresponding FDs are in a large range $5-15 \%$. Figure 8 shows the plots for six largest FDs in the past cycles 19-22. Their six pairs of Dst and FD are plotted in Fig. 6 as triangles. These are in a large range of Dst $(-105$ to -325$)$. The correlation for the 12 events (open circles and triangles only) was negative $(-0.70)$, indicating as if large FDs avoided large Dst and vice versa, but this is an illusion. The overall correlation for all the twenty nine pairs in Fig. 6 is -0.17 , indicating a gross 

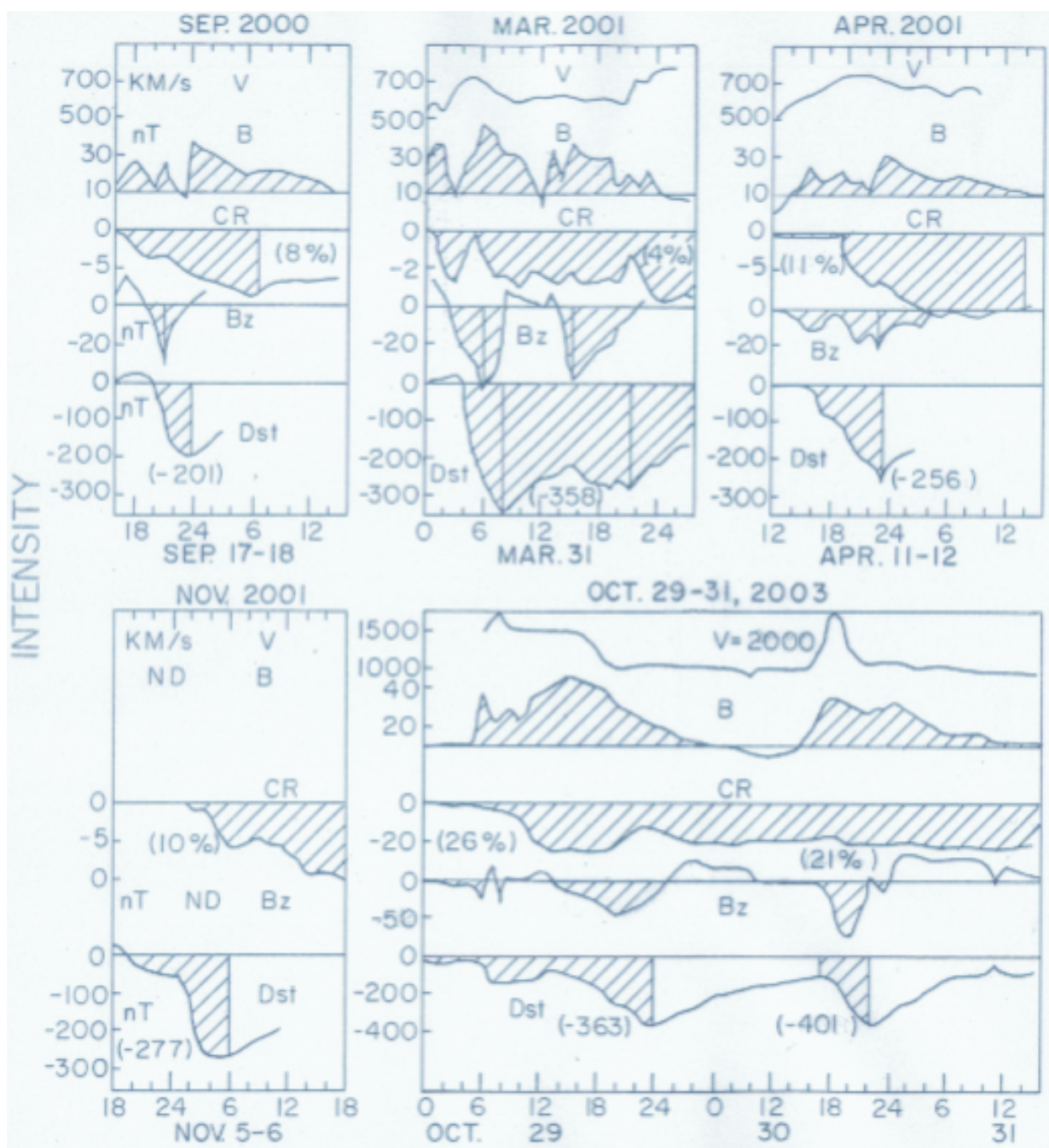

OCT. $29-31,2003$

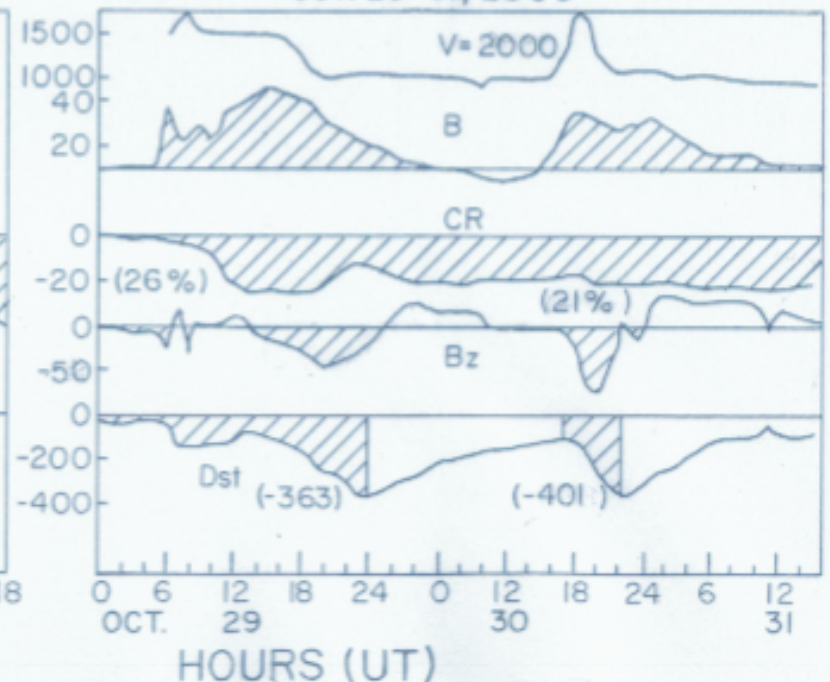

Fig. 3. Same as Fig. 1, for the six events 17-18 September 2000, 31 March 2001, 11-12 April 2001, 5-6 November 2001, 29 October 2003 and 30 October 2003.

Table 3. Details of the six largest Dst events and six largest FDs in cycles 19-22.

\begin{tabular}{lccclccc}
\hline \multicolumn{2}{c}{ Largest Dst } & Dst(nT) & CR(\%) & \multicolumn{2}{c}{ Largest FDs } & CR(\%) & Dst(nT) \\
\hline 13 Mar & 1989 & -589 & -15 & 15 Feb & 1978 & -26 & -108 \\
15 Jul & 1959 & -429 & -15 & 4 Aug & 1972 & -28 & -125 \\
13 Sep & 1957 & -427 & -5 & 24 Mar & 1991 & -18 & -298 \\
11 Feb & 1958 & -426 & -6 & 13-14 Jul & 1982 & -19 & -325 \\
25-26 May & 1967 & -387 & -7 & 28 Oct & 1991 & -16 & -196 \\
8-9 Nov & 1991 & -354 & -9 & 20-21 Oct & 1989 & -17 & -268 \\
\hline
\end{tabular}

dissimilarity between the magnitudes of Dst and the corresponding FDs (statistically, almost no relationship).

\section{Origin of Dst through additional mechanisms}

The Dst obtained from data of ground magnetometers is partly due to the ring current, and some contributions come from other sources also (Gonzalez et al., 1994). Thus, there 


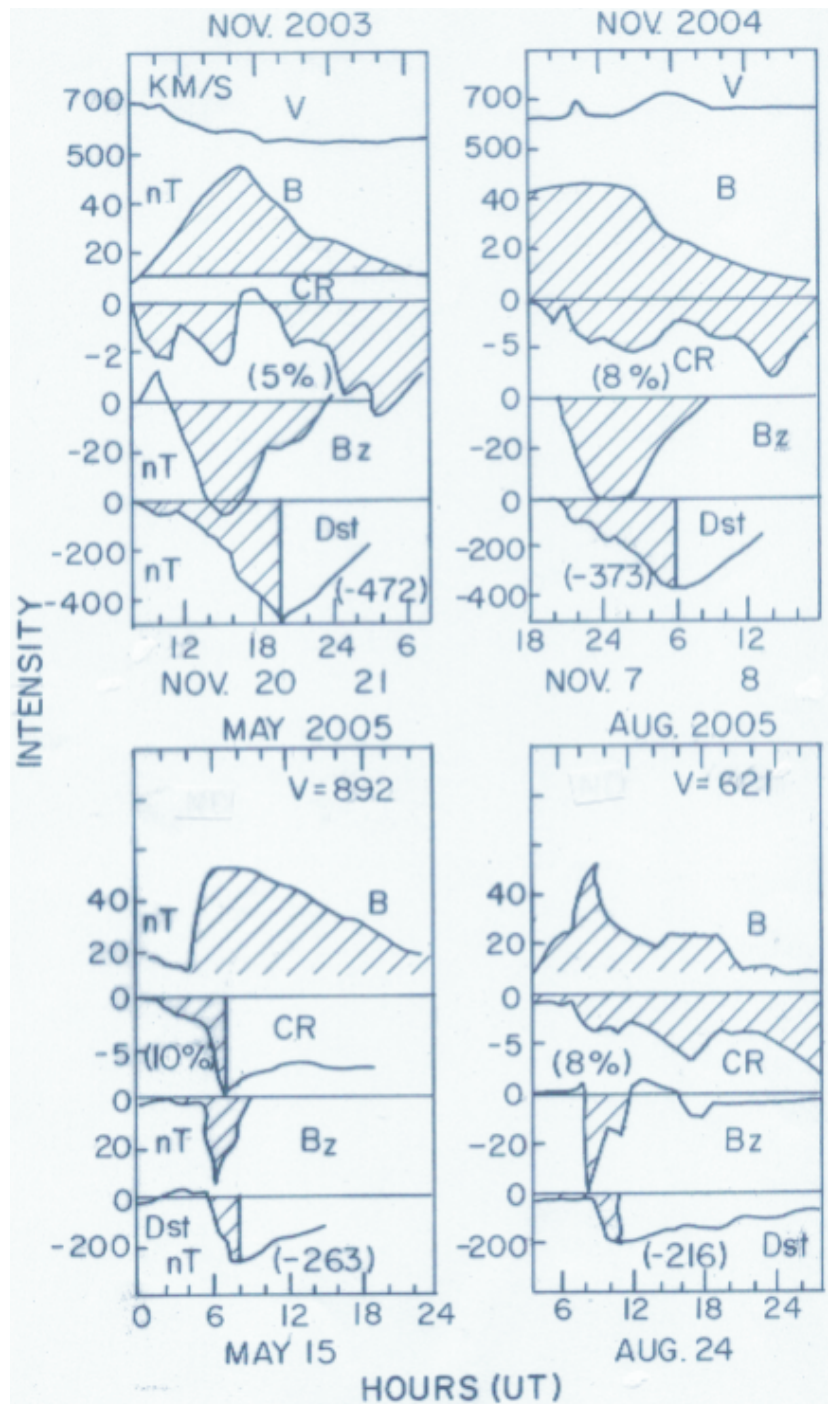

Fig. 4. Same as Fig. 1, for the four events, 20-21 November 2003, 7-8 November 2004, 15 May 2005 and 24 August 2005.

are induced currents in the solid Earth. These are mostly in a constant proportion with the ring current (about one third) and hence will not affect the correlations with $B_{z}$ (only the correlation coefficient will alter). But there are magnetopause currents which depend upon the solar wind ram pressure. Their contribution to Dst can be substantial and may differ considerably from event to event, even though $B_{z}$ may remain the same. For the true ring current effect Dst* after a correction due to magnetopause currents is applied to Dst, Gonzalez et al. (1994) mention the relationship between Dst and Dst* as

Dst $*=\mathrm{Dst}-b p^{1 / 2}+c$

where $p$ is the storm time solar wind ram pressure, obtained as $\mathrm{Nm}^{+} V^{2}$ ( $N$ and $V$ being the solar wind density and speed, respectively and $m^{+}$the proton mass), $b$ is a proportional-

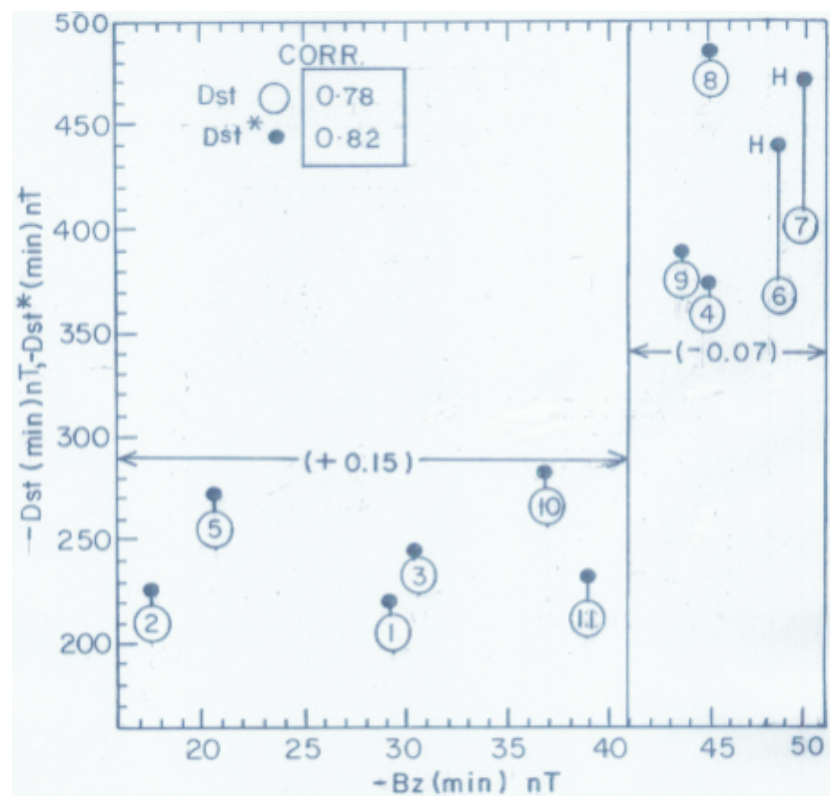

Fig. 5. Plots of maximum negative magnitudes of Dst versus (a) maximum negative magnitudes of $B_{z}$, (b) $B_{z}(\mathrm{CP})$, (c) $V B_{z}$ and (d) $V B_{z}(\mathrm{CP})$. Corresponding correlations are indicated.

ity factor, and $c$ is the quiet time solar wind ram pressure contribution. Typically, $b=0.2 \mathrm{nT} /\left(\mathrm{ev} \mathrm{cm}^{-3}\right)^{1 / 2}$ and $c=20 \mathrm{nT}$. In our Table 2, the $N, V$ values are available only for nine events. Using those values, the Dst* was calculated and correlated with maximum negative $B_{z}$. It gave a high correlation of 0.70 , in contrast to the 0.28 between Dst and negative $B_{z}$. Also, for cumulative (partial) $B_{z}$, namely $B_{z}(\mathrm{CP})$, the Dst* gave a correlation 0.83 in contrast to the 0.59 between Dst and $B_{z}(\mathrm{CP})$. (Correlations given in Table 4). Thus, magnetopause currents can make a substantial contribution to ground Dst (tens of nT, even exceeding $100 \mathrm{nT}$ for very severe events), and when taken into consideration, the relationship between the ring current Dst* and negative $B_{z}$ is almost perfect as envisaged in the Dungey (1961) mechanism. Incidentally, the correlation of Dst* with $V B_{z}$ was only 0.40 in contrast to the 0.59 between Dst and $V B_{z}$. We feel that the proposed $V B_{z}$ mechnism is illusionary and unrealistic, and the effect of $V$ is felt only through the ram pressure and the subsequent magnetopause current contribution to Dst. If true, all the results about Dst relationship with $B_{z}$ reported in the literature in the past need reassessment after taking into account the magnetopause current contribution. One would think that since moderate and weak Dst storms are caused by weaker interplanetary structures where $N$ and $V$ are smaller, the ram pressure would be small and the magetopause current contributions to Dst would be small. True, but since the Dst also is smaller, the percentage contribution of magnetopause currents to Dst may still be substantial. Hence, correction for magnetopause currents will be necessary in all cases. 
Table 4. Correlations of Dst and Dst* with other parameters.

\begin{tabular}{lccccccccc}
\hline & $B_{z}$ & $\mathrm{Cum} B_{z}(\mathrm{P})$ & $\mathrm{Cum} B_{z}(\mathrm{~T})$ & $B$ & $\mathrm{CR}(\%)$ & $V B_{z}$ & $V B_{z}(\mathrm{CP})$ & $N V B_{z}$ & $N V B_{z}(\mathrm{CP})$ \\
\hline Dst & 0.28 & 0.59 & 0.48 & 0.50 & 0.31 & 0.59 & 0.71 & 0.25 & 0.68 \\
Dst* & 0.70 & 0.83 & 0.65 & 0.47 & 0.11 & 0.40 & 0.77 & 0.09 & 0.57 \\
\hline
\end{tabular}

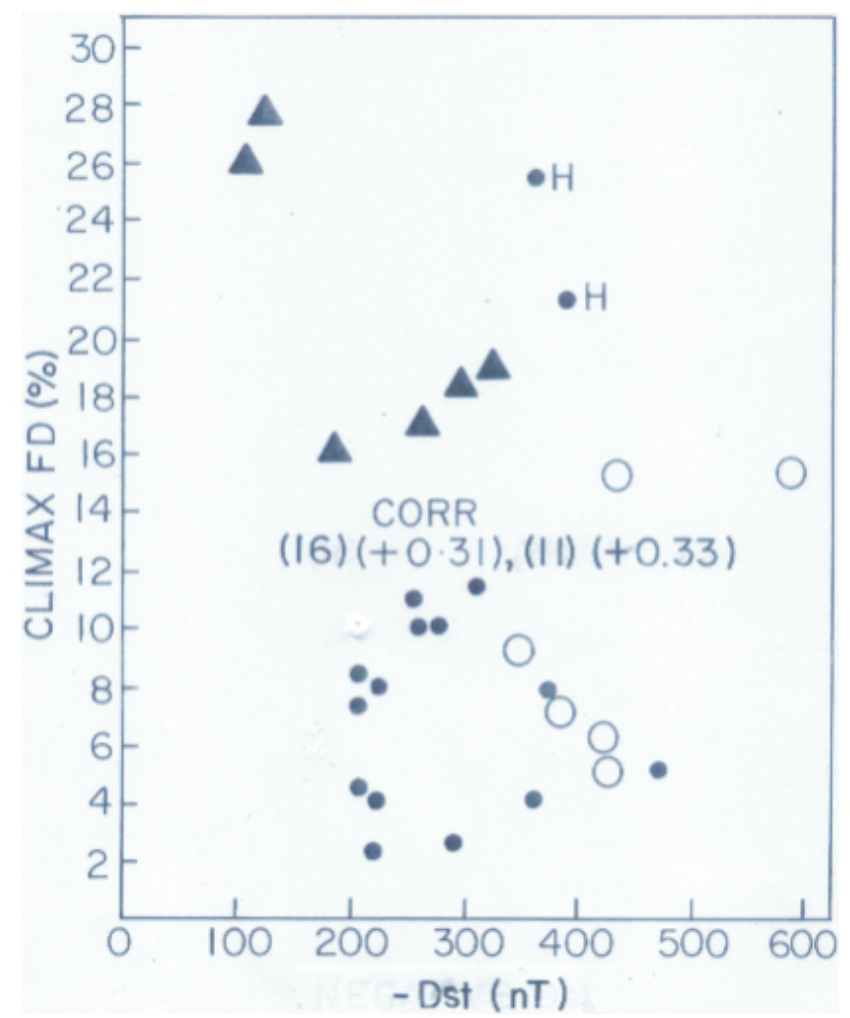

Fig. 6. Plot of Climax FD magnitudes versus maximum negative Dst for seventeen severe Dst events in cycle 23 (dots), for six largest Dst events in cycles 19-22 (open circles) and for six largest FDs in cycles 19-22 (triangles).

Belov et al. (2005) mention that during the storm of November 2003, there was an increase of cosmic ray intensity of $7 \%$ in the Athens neutron monitor inside the Forbush decrease and attribute it to magnetospheric effects. In the present work, such effects are not considered and only FD with respect to the pre-storm level is estimated.

\section{Conclusions and discussion}

1. Severe storms and Forbush decreases during cycle 23 showed that maximum negative Dst magnitudes usually occurred almost simultaneously with the maximum negative values of the $B_{z}$ component of interplanetary mag- netic field $\boldsymbol{B}$, but the maximum magnitudes of negative Dst and $B_{z}$ were poorly correlated $(+0.28)$.

2. A parameter $B_{z}(\mathrm{CP})$ was calculated (cumulative partial $B_{z}$ ) as sum of the hourly negative values of $B_{z}$ from the time of start to the maximum negative value. The correlation of negative Dst maximum with $B_{z}(\mathrm{CP})$ was higher $(+0.59)$ as compared to that of Dst with $B_{z}$ alone $(+0.28)$.

3. When the product of $B_{z}$ with the solar wind speed $V$ (at the hour of negative $B_{z}$ maximum) was considered, the correlation of negative Dst maximum with $V B_{z}$ was +0.59 and with $V B_{z}(\mathrm{CP}), 0.71$. Thus, including $V$ improved the correlations.

4. Ground Dst has a considerable contributions from the magnetopause currents produced by the solar wind ram pressure (a function of solar wind number density $N$ and speed $V$ ). To get a correct estimate of the true ring current Dst*, the magnetopause contribution needs to be subtracred from Dst. The Dst* shows much better correlations with $B_{z}$ and cumulative $B_{z}(\mathrm{CP})$, than Dst does.

5. Maximum negative Dst did not occur at the same hour as maximum FD. The time evolutions of Dst and FD were very dissimilar.. The correlations were almost zero. (The correlation with Dst* with FD was not any better, it was only 0.11). When extreme events in earlier cycles 19-22 (six largest Dst and six largest FDs) were considered, the correlation between Dst and FD for the twelve events was negative $(-0.70)$ giving an impression that large Dst avoided large FDs and vice versa. However, this could be an illusion. Basically, all that it means is that negative Dst and FDs are uncorrelated. All this needs further confirmation with future data.

6. In some events, FDs started when interplanetary total magnetic field $\boldsymbol{B}$ (as measured at the satellite) started rising, but their later evolutions were dissimilar (one having two humps, other not, etc.). During cycle 23, the correlation between maximum FD and maximum $B$ (not necessarily at the same hour) was low $(-0.18)$ but the correlation with solar wind speed $V$ was high (0.90). Since cosmic rays have very high speeds (almost $300000 \mathrm{~km} \mathrm{~s}^{-1}$ ) as compared to solar wind speeds 


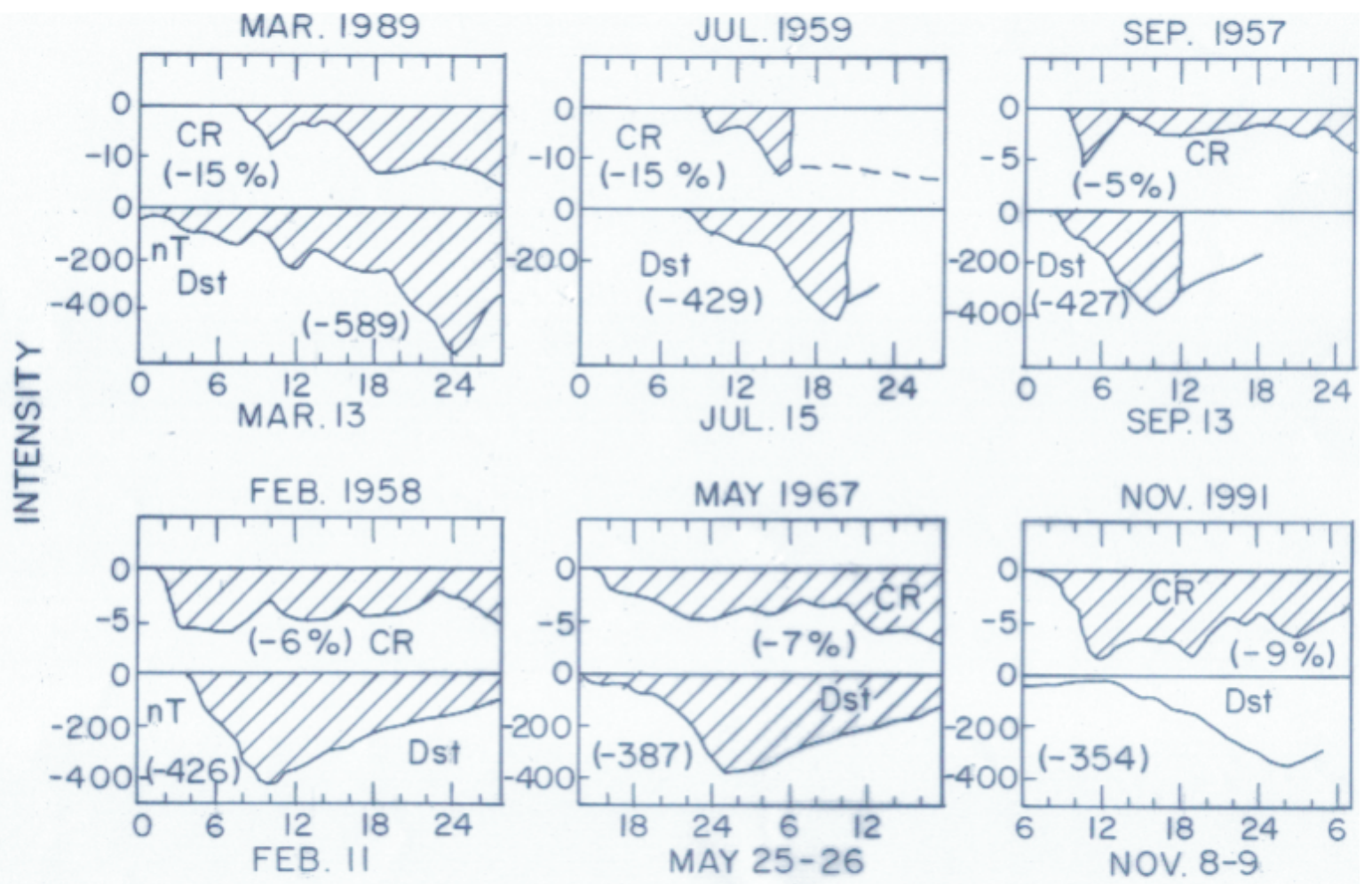

Fig. 7. Plots of hourly values of CR depressions (FDs) and geomagnetic Dst for the six largest Dst events 13 March 1989 , 15 July 1959 , 13 September 1957, 11 February 1958, 25-26 May 1967 and 8-9 November 1991.

(2500 $\mathrm{km} \mathrm{s}^{-1}$ or less), a direct effect of $V$ on FD is unlikely. Indirectly, very strong interplanetary structures have very high speeds and also very well organized large magnetic field $(\boldsymbol{B})$ structures, which can deviate cosmic ray trajectories effectively and cause modulations (FDs). In this scenario, strong FDs imply very large interplanetary blobs, which also would have large negative $B_{z}$, which would result in large Dst storms also, except that sometimes, the $B_{z}$ component is wavering (fluctuating) between negative and zero (or even positive) values and the ring current formation is partly interrupted, yielding Dst values not very high. The most famous example of such a case is that of the 4 August 1972 event (Fig. 8) where the Coronal Mass Ejection had a record transit average velocity of $\sim 2800 \mathrm{~km} \mathrm{~s}^{-1}$ (transit time $<15 \mathrm{~h}$, see Kane, 2005; Tsurutani et al., 2003), which should have resulted in an enormous Dst, but, as it happened, the $B_{z}$ component wavered considerably and the resulting Dst was a paltry $108 \mathrm{nT}$. Thus, large FDs are a consequence of deviations of cosmic ray trajectories in large, smooth interplanetary magnetic field $\boldsymbol{B}$ structures, while large Dst is caused only when $B_{z}$ is large negative in a very small region in the magetotail. This happens often when the main blob has high speeds and high $\boldsymbol{B}$, but not invariably and in those few cases, Dst storms are not as large as expected. All this needs further confirmation with future data.
The lack of a good correlation between CR decreases and $\boldsymbol{B}$ is not surprising. Wibberenz et al. (1998) mention specifically that Forbush decreases occur in two steps (the first one heralding entry into a shock and the second one, entry into the ejecta) and their dependence on energy, time and spatial position of the observer is different. Thus, the component related to the shock shows generally a gradual decrease and a slow recovery (lasting up to eight days). The ejecta component lasts typically one day (a very localized effect) and, having a small longitudinal extent, may even miss the spacecraft. On the average, when both shocks and ejecta are seen, the contributions to the CR depression of shock and ejecta are roughly equal (half-half), but in individual cases, due to large variability of the ejecta effect, the contributions may be unequal, and sometimes, only one (shock or ejecta) may account for the total effect. For the "shock effect", in a simple model, Wibberenz et al. (1998) show that the CR decrease would depend upon the thickness $L$ of the barrier and the variations of wind speed $V$, magnetic field $\boldsymbol{B}$ and radial diffusion coefficient $K$ across the shock front. There is no way to estimate the diffusion coefficient. The "ejecta effect" have been discussed by Cane et al. (1995) in terms of a simple model in which particles gain entry to the ejecta via perpendicular diffusion. The FD magnitude is a function of the product VB, the radius $a$ of the magnetic cloud, and the distance $r$ from the Sun. However, the dependence of FD on the interplanetary parameters is different for the 

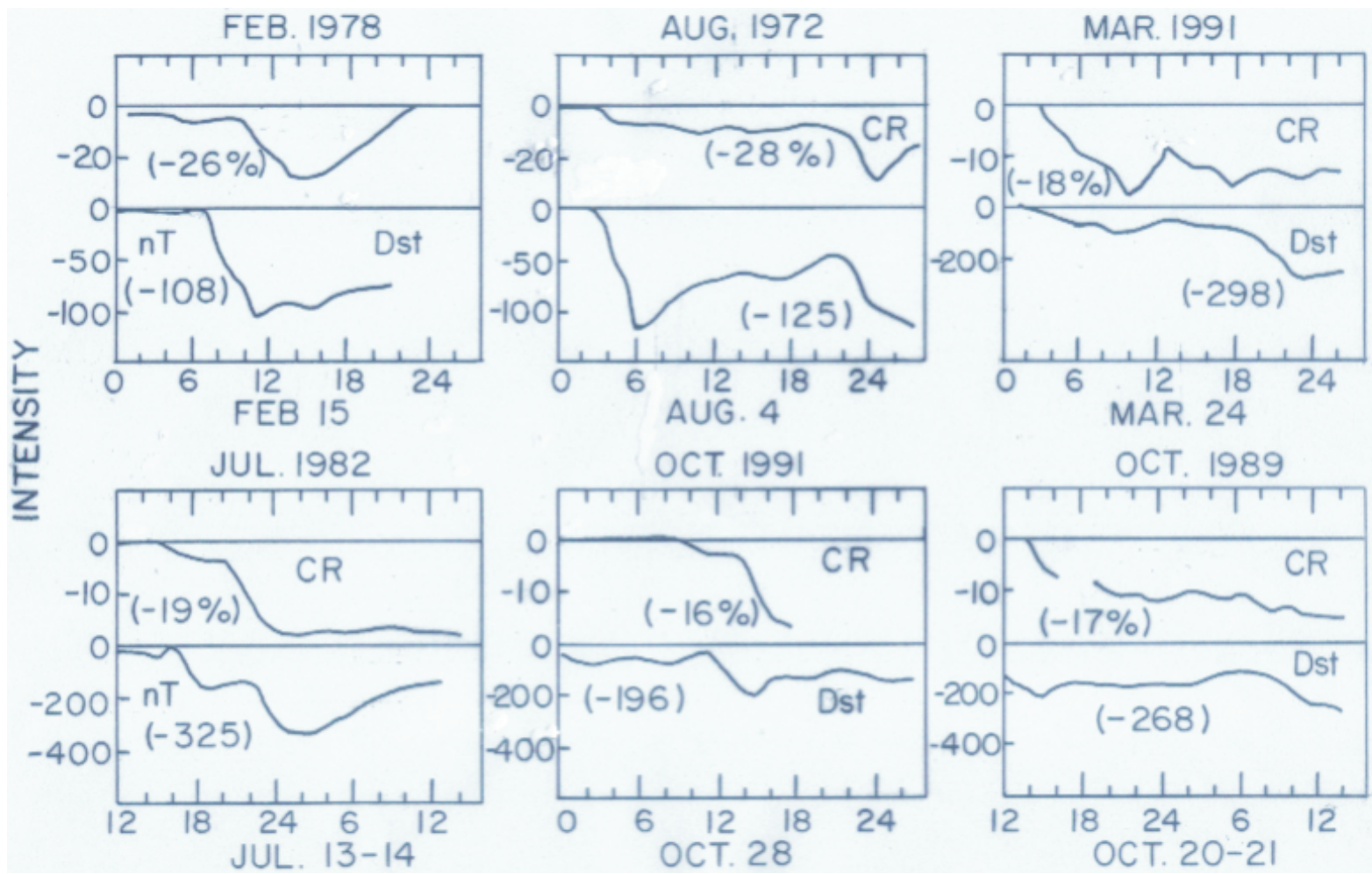

Fig. 8. Same as Fig. 7, for the six largest FDs 15 February 1978, 4 August 1972, 24 March 1991, 13-14 July 1982,28 October 1991 and 20-21 October 1989.

"shock effect" and the "ejecta effect". Hence, Wibberenz et al. (1998) suggest that a detailed study where the amplitudes (separately for the shock and ejecta) are related to the interplanetary parameters of individual events would be more useful than the definition of an "average" event. Interplanetary $V$ and $B$ can be measured directly (though only as snapshots at the satellite location), but the radial diffusion coefficient $K$ cannot be measured directly and has to be estimated indirectly (Morfill and Scholer, 1977, estimated a decrease of $K$ of approximately one order of magnitude during a storm). Here, we are only illustrating the looseness of the FD- $V B$ relationship, and the looseness could be due to the lack of knowledge of the diffusion coefficient. Incidentally, the model of Wibberenz et al. (1998) considers the ratios of $V$ and $B$, during and before the event. Thus, not the actual values of increased $V$ and $B$ are to be used but their ratios with their pre-storm values. In our plots, the FD main phase does not seem to have a two step structure. We attempted correlations with $(V-V o),(V / V o),(B-B o)$ and $(B / B o)$ and their combinations, but the correlations did not improve. An additional complication is that the FD effect would be largest if the Earth passes very near the center of the interplanetary blob. If the center is above or below the plane of Earth's orbit around the Sun, the path of Earth will not see a full FD effect even though the $B$ value may be large.
One would have thought that some more information about interplanetary structures could be obtained by examining them by multiple satellites. However, as mentioned by Cane (2000), such an effort may not very useful because, besides other complications, the interplanetary structures evolve with time and distance from the Sun and are altered considerably when they reach different satellites at different distances from the Sun. However, data from multiple satellites have their other uses.

In the present work, only data for one location (Climax) are used for cosmic ray FD. Are such data fully representative of cosmic ray effects? The question arises because firstly, cosmic rays bend in magnetic fields and have trajectories depending upon the cut-off rigidities at any geographical location (lesser the cut-off rigidity, larger the FD, because more low energy particles are received). Secondly, the trajectories at different longitudes may sample different parts of the interplanetary shocks, which may not be uniform. In a rigorous analysis, the trajectories for data at different locations needs to be examined, which is beyond the scope of the present work. However, it should be kept in mind that using cosmic ray data at only one location may involve uncertainties of a few percent in the FD estimates. We think that any results reported here would still be true with uncertainties of this order. 
Acknowledgements. This work was partially supported by FNDCT, Brazil, under contract FINEP-537/CT.

Topical Editor I. A. Daglis thanks H. Mavromichalaki and A. Belov for their help in evaluating this paper.

\section{References}

Barnden, L. R.: The Large-Scale Magnetic Field Configuration Associated With Forbush Decreases, Proc. 13th Int. Cosmic Ray Conf., 2, 1277, 1973.

Belov, A. V. and Ivanov, K. G.: Forbush-Effects in 1977-1979, Proc. 25th Int. Cosmic Ray Conf., Durban, 1, 421, 1997.

Belov, A. V., Eroshenko, E. A., Oleneva, V. A., Struminsky, A. B., and Yanke, V. G.: What determines the magnitude of Forbush decreases?, Adv. Space Res., 27(3), 625-630, 2001.

Belov, A., Baisultanova, L., Eroshenko, E., Mavromichalaki, H., Yanke, V., Pchelkin, V., Plainaki, C., and Mariatos, G.: Magnetospheric effects in cosmic rays during the unique magnetic storm on November 2003, J. Geophys. Res., 110, A09S20, doi:10.1029/2005JA011067, 2005.

Burlaga, L. F., McDonald, F. B., Ness, N. F., Schwenn, R., Lazarus, A. J., and Mariani, F.: Interplanetary flow systems associated with cosmic ray modulation in 1977-1980, J. Geophys. Res., 89, 6579-6587, 1984.

Cane, H. V.: Cosmic Ray Decreases and Magnetic Clouds, J. Geophys. Res., 98, 3509-3512, 1993.

Cane, H. V.: Coronal mass ejections and Forbush decreases, Space Sci. Rev., 93, 55-77, 2000.

Cane, H. V., Richardson, I. G., and Wibberenz, G.: The Response of Energetic Particles to the Presence of Ejecta Material, Proc. 24th Int. Cosmic Ray Conf., 4, 377, 1995.

Cane, H. V., Richardson, I. G., and von Rosenvinge, T. T.: Cosmic ray decreases: 1964-1994, J. Geophys. Res., 101, 21561-21572, 1996.

Dungey, J. W.: Interplanetary magnetic field and the auroral zones, Phys. Rev. Lett., 6, 47-48, 1961.

Forbush, S. E.: On the Effects in the Cosmic-Ray Intensity Observed During the Recent Magnetic Storm, Phys. Rev., 51, 11081109, 1937.

Gonzalez, W. D. and Echer, E.: A study on the peak Dst and peak negative $B_{z}$ relationship during intense geomagnetic storms, Geophys. Res. Lett., 32, L18103. doi:10.1029/2005GL023486, 2005.

Gonzalez, W. D., Joselyn, J. A., Kamide, Y., Kroehl, H. W., Rostoker, G., Tsurutani, B. T., and Vasyliunas, V.: What is a geomagnetic storm?, J. Geophys. Res., 99, 5771-5792, 1994.

Hess, V. F. and Demmelmair, A.: World-wide Effect in Cosmic Ray Intensity. as Observed During a Recent Geomagnetic Storm, Nature, 140, 316-317, 1937.
Iucci, N., Parisi, M., Storini, M., and Villoresi, G.: Forbush Decreases: Origin and Development in the Interplanetary Space, Nuovo Cimento, 2C, 1-52, 1979a.

Iucci, N., Parisi, M., Storini, M., and Villoresi, G.: High Speed Solar Wind Streams and Galactic Cosmic Ray Modulation, Nuovo Cimento, 2C, 421-438, 1979b.

Iucci, N., Pinter, S., Parisi, M., Storini, M., and Villoresi, G.: The Longitudinal Asymmetry of the Interplanetary Perturbation Producing Forbush Decreases, Nuovo Cimento, 9C, 39-50, 1986.

Kane, R. P.: A comparative study of geomagnetic, interplanetary, and cosmic ray storms, J. Geophys. Res., 82, 561-577, 1977.

Kane, R. P.: How good is the relationship of solar and interplanetary plasma parameters with geomagnetic storms?, J. Geophys. Res., 110, A02213, doi:10.1029/2004JA010799, 2005.

Kudela, K. and Brenkus, R.: Cosmic ray decreases and geomagnetic activity: list of events 1982-2002, J. Atmos. Solar-Terr. Phys., 66, 1121-1126, 2004.

Lockwood, J. A.: Forbush Decreases in the Cosmic Radiation, Space Sci. Revs., 12, 658-715, 1971.

Morfill, G. E. and Scholer, M.: Influence of interplanetary shocks on solar particle events, Astrophys. Space Sci., 46, 73-86, 1977.

Nagashima, K., Sakakibara, S., Fujimoto, K., Tatsuoka, R., and Morishita, I.: Localized Pits and Peaks in Forbush Decrease, Associated with Stratified Structure of Disturbed and Undisturbed Magnetic Fields, Nuov. Cimento, 13C, 551-587, 1990.

Papaioannou, A., Belov, A., Mavromichalaki, H., Eroshenko, E. and Oleneva, E. V.: The rare exclusion of the July 2005 cosmic ray variations resulted from western and behind the limb solar activity, Adv. Space Res., 43, 582-588, 2009.

Papaioannou, A., Mavromichalaki, H., Eroshenko, E., Belov, A., and Oleneva, V.: The burst of solar and geomagnetic activity in August-September 2005, Ann. Geophys., 27, 1019-1026, 2009, http://www.ann-geophys.net/27/1019/2009/.

Simpson, J. A.: Cosmic-Radiation Intensity-Time Variations and Their Origin. III The Origin of 27-Day Variations, Phys. Rev., 94, 426-440, 1954.

Skoug, R. M., Gosling, J., Steinberg, J., McComas, D. J., Smith, C. W., Ness, N. F., Hu, Q., and Burlaga, L. F.: Extremely high speed solar wind: October 29-30, 2003, J. Geophys. Res., 109, A09102, doi:10.1029/2004JA010494, 2004.

Sugiura, M.: Hourly values of equatorial Dst for the IGY, Ann. Int. Geophys. Year, 35, 945-948, Pergamon Press, Oxford, 1964.

Tsurutani, B. T., Gonzalez, W. D., Lakhina, G. S., and Alex, S.: The extreme magnetic storm of 1-2 September 1859, J. Geophys. Res., 108(A7), 1268, doi:10.1029/2002JA009504, 2003.

Wibberenz, G., le Roux, J. A., Potgieter, M. S., and Bieber, J. W.: Transient Effects and Disturbed Conditions, Space Sci. Rev., 83, 309-348, 1998. 